\title{
Potencialidades e fragilidades de implantação da Política Nacional de Práticas Integrativas e Complementares
}

\author{
Implementation of Brazil's National Policy on Complementary \\ and Integrative Practices: strengths and weaknesses
}

Pedro Henrique Leonetti Habimorad (https://orcid.org/0000-0001-9276-6019) ${ }^{1}$ Fernanda Martin Catarucci (https://orcid.org/0000-0003-2935-2475) ${ }^{1}$

Vânia Hercília Talarico Bruno (https://orcid.org/0000-0002-5101-5435) ${ }^{1}$

Ivan Beteto da Silva (https://orcid.org/0000-0003-2805-0192) ${ }^{1}$

Violeta Campolina Fernandes (https://orcid.org/0000-0001-5549-072X) ${ }^{1}$

Marcelo Marcos Piva Demarzo (https://orcid.org/0000-0002-7447-1839) ${ }^{2}$

Regina Stella Spagnuolo (https://orcid.org/0000-0002-6977-4165) ${ }^{1}$

Karina Pavão Patricio (https://orcid.org/0000-0003-2112-5956) ${ }^{1}$
${ }^{1}$ Faculdade de Medicina, Universidade Estadual Paulista Júlio de Mesquita Filho. Av. Prof. Mário Rubens Guimarães Montenegro $\mathrm{s} / \mathrm{n}$ Campus de Botucatu. 18618-687 Botucatu SP Brasil.peuhabimorad@ hotmail.com

${ }^{2}$ Escola Paulista de Medicina, Universidade Federal de São Paulo. São Paulo SP Brasil.

\begin{abstract}
This narrative review examines the literature on complementary and integrative practices (CIPs) and their incorporation into Brazil's national health system (Sistema Único de Saúde - SUS) in an attempt to understand the strengths and weaknesses of the implementation of the $\mathrm{Na}$ tional Policy on Complementary and Integrative Practices in the SUS (PNPIC, acronym in Portuguese). A search was conducted of the MEDLINE, LILACS, and SciELO databases, resulting in final sample of 25 articles. Our analysis identified five key themes in the literature related to the strengths and weaknesses of policy implementation: 1) Professional training in CIPs in the SUS; 2) structuring the provision of CIPs, access, and health promotion; 3) knowledge, access, and acceptance of service users in relation to CIPs; 4) knowledge of SUS professional staff and managers in relation to the PNPIC; and 5) scope and monitoring and evaluation of the PNPIC. In consonance with the conclusions of the PNPIC management reports, the findings provide a deeper insight into policy implementation problems and reinforce the need to empower the actors involved in this process to tackle these challenges.

Key words Review, Complementary Therapies, Unified Health System, Public Policy
\end{abstract}

Resumo Esta revisão narrativa tem por objetivo analisar a produção científica sobre as Práticas Integrativas e Complementares (PIC) no Sistema Unico de Saúde (SUS) visando compreender as potencialidades e fragilidades do processo de implantação da Politica Nacional de Práticas Integrativas e Complementares (PNPIC). Após busca nas bases de dados, 25 artigos foram selecionados $e$ os seus resultados analisados criticamente. $\mathrm{Da}$ análise do material emergiram cinco temas principais que explicitaram potencialidades e fragilidades de implantação da política: 1) Formação profissional em PIC para o SUS; 2) Estruturação da oferta em PIC, acesso e promoção da saúde; 3) Conhecimento, acesso e aceitação de usuários em relação às PIC; 4) Conhecimento de profissionais e gestores em relação à PNPIC; e 5) Escopo, monitoramento e avaliação da PNPIC. Os resultados se alinham aos relatórios de gestão da PNPIC aprofundando o conhecimento acerca da implantação da política e reforçando a necessidade de empoderamento dos atores do SUS para o enfrentamento de seus desafios.

Palavras-chave Revisão, Terapias Complementares, Sistema Único de Saúde, Política Pública 


\section{Introdução}

Diante do cenário de transição epidemiológica do século XX, no qual as doenças crônicas e degenerativas emergem como principal causa de morbimortalidade mundial inicia-se uma mudança de paradigma na saúde pública na qual a Atenção Primária em Saúde (APS), a prevenção e a promoção de saúde revelam-se como estratégias prioritárias ${ }^{1}$.

A partir da década de 70 outros determinantes da saúde das populações (biológicos, ambientais e de estilo de vida) passam a ganhar mais relevância levando a questionamentos em relação à eficácia da biomedicina como único modelo de atenção à saúde pública. Este contexto, somado ao alto custo das ações biomédicas, ocasiona um crescente interesse da sociedade ocidental pelas práticas terapêuticas que não pertencem à medicina científica.

As Práticas Integrativas e Complementares, por privilegiarem a atividade terapêutica e se basearem em teorias voltadas para os aspectos ambientais e comportamentais do processo saúde-doença, caracterizam-se como estratégias potencialmente interessantes para o enfrentamento dos novos desafios na atenção à saúde.

Por esta razão, a Organização Mundial da Saúde (OMS) lança na década de 70 o Programa de Medicina Tradicional, visando o desenvolvimento de políticas públicas na área. Em 2002, é publicado um segundo documento WHO Traditional Medicine Strategy ${ }^{2}$ que legitima o primeiro, visando apoiar programas nacionais de pesquisa e treinamento, definir diretrizes técnicas e padrões internacionais, facilitar a troca de informações e integrar a Medicinas Tradicionais/Medicinas Alternativas e Complementares (MT/MCA) nos sistemas nacionais de saúde.

Em virtude das demandas apresentadas nas Conferências Nacionais de Saúde (CNS) e nas recomendações da OMS, o Ministério da Saúde (MS) aprovou em 2006 os documentos: Política Nacional de Plantas Medicinais e Fitoterápicos ${ }^{3} \mathrm{e}$ Política Nacional de Práticas Integrativas e Complementares no SUS (PNPIC) ${ }^{4}$, que norteiam o desenvolvimento das PIC no SUS.

Segundo a PNPIC, a incorporação dessas práticas na saúde pública se justifica principalmente pela garantia do princípio da integralidade. Neste sentido, sua principal finalidade é atender a necessidade de conhecer, apoiar e incorporar as experiências com PIC já em andamento na rede pública de saúde visando estimular os mecanismos naturais de prevenção, recuperação e promoção da saúde, com ênfase na APS ${ }^{4}$.
Dois anos após a publicação da PNPIC foi realizado um diagnóstico, em parceria com o Departamento de Atenção Básica (DAB) com o objetivo de analisar o conhecimento, a institucionalização e a oferta nas PIC nos municípios brasileiros ${ }^{5}$. A partir dos resultados deste trabalho, foram listados alguns desafios mais imediatos e estratégicos para o desenvolvimento da política: 1) viabilização da formação e qualificação dos profissionais em número adequado para atuar no SUS; 2) implementação do Monitoramento e Avaliação; 3) o fornecimento de insumos (medicamentos homeopáticos/fitoterápicos, agulhas para MTC/acupuntura); e 4) a implementação de pesquisa em PIC, fomentando a ampliação de conhecimento, considerando as necessidades e diretrizes do SUS.

Em 2011, a Coordenação Nacional de Práticas Integrativas e Complementares, publicou o relatório de Gestão 2006-2010 . O documento traz um panorama da PNPIC no SUS no âmbito da APS. O principal avanço relatado neste documento refere-se à normatização e institucionalização das experiências em PIC nos municípios, em consonância com as diretrizes do documento da OMS. Como perspectivas, destaca-se a criação do Núcleo de Apoio a Saúde da Família (NASF) que viabiliza a inserção de profissionais médicos homeopatas e acupunturistas, bem como outros profissionais praticantes de PIC no âmbito da Atenção Básica. Como desafios o relatório resgata aqueles apontados pelo primeiro diagnóstico e acrescenta outros como a estruturação dos serviços na rede pública e o desenvolvimento/adequação de legislação específica para os serviços no SUS.

Após 11 anos da implantação da PNPIC, o MS publicou em 2017 a Portaria no 849/2017 que amplia os procedimentos oferecidos pela política no SUS. A meditação, arteterapia, musicoterapia, tratamento naturopático, tratamento osteopático, tratamento quiroprático e reiki passam a integrar a oferta de PIC. A terapia comunitária, dança circular/biodança, yoga, oficina de massagem/automassagem, auriculoterapia, massoterapia, tratamento termal/crenoterápico já faziam parte dos serviços desde abril de 2016. Todos esses procedimentos, por meio de dados confirmados pelo Programa Nacional de Melhoria do Acesso e da Qualidade na Atenção Básica (PMAQ-AB) eram realizados por muitos municípios brasileiros.

Em 2018, na ocasião do $1^{\circ}$ Congresso Internacional de Práticas Integrativas e Complementares e Saúde Pública, o MS anunciou a inclusão 
de outras dez práticas no SUS, através da Portaria $n^{\circ} 702 / 2018^{8}$, que altera a anterior. Foram incluídas as seguintes PIC: aromaterapia, apiterapia, bioenergética, constelação familiar, cromoterapia, geoterapia, hipnoterapia, imposição de mãos, ozonioterapia e terapia de florais. A medicina antroposófica e a crenoterapia, que tinham sido incluídas em 2016 como observatório, foram definitivamente incluídas em 2018.

Tendo em vista mais de uma década de publicação da PNPIC, surge a inquietação: o que a literatura científica sobre as PIC no SUS aponta em relação à implantação desta política?

O presente estudo tem por objetivo revisar e analisar as produções científicas sobre esta temática, procurando identificar os principais temas abordados, as fragilidades e potencialidades de implantação da PNPIC.

\section{Métodos}

Trata-se de uma revisão narrativa da literatura caracterizada por Rother ${ }^{9}$ como um estudo apropriado para descrever e discutir o "estado da arte" de um determinado assunto, de um ponto de vista teórico ou contextual.

Diferentemente das revisões sistemáticas, a revisão narrativa parte de questões amplas visando uma síntese qualitativa da literatura do ponto de vista crítico do autor. Neste sentido, tem a finalidade de atualizar o leitor sobre um tema cientificamente relevante, propor novas questões e contribuir para o seu debate 9 .

A coleta de dados ocorreu entre Julho e Dezembro de 2016 nas bases de dados Medline, Lilacs e Scielo, utilizando os seguintes descritores: Medicina Integrativa (e descritores sinônimos) e Sistema Único de Saúde.

Inicialmente foram encontrados 222 resultados (198 na Lilacs, 4 no Medline e 20 na Scielo). Durante a seleção dos artigos, que ocorreu mediante a leitura dos títulos e resumos, foram aplicados os seguintes critérios de inclusão: artigos completos e disponíveis, publicados em português, inglês ou espanhol, no período entre 2006 e 2016 (que compreende um período de dez anos desde a criação da política), que abordavam pesquisas qualitativas e/ou quantitativas, realizadas com atores ou com potenciais atores do SUS (profissionais, usuários, gestores e estudantes da área da saúde).

Dado que a revisão narrativa não visa esgotar as fontes, considerou-se suficiente o número de artigos encontrados que cumpriram com os critérios de inclusão optando-se pela exclusão de comunicações, editoriais, entrevistas, revisões de literatura, monografias, teses, dissertações e trabalhos repetidos nas bases de dados.

Desta forma, a amostra final foi composta por 25 artigos. Após a seleção dos artigos (Figura 1), procedeu-se a leitura e análise crítica do material. A revisão narrativa foi organizada em cinco temas que foram discutidos à luz da literatura teórica sobre as PIC no SUS e sobre a integralidade.

\section{Resultados e Discussão}

O Quadro 1 sintetiza os principais temas que emergiram da análise dos artigos, explicitando as principais potencialidades e fragilidades de implantação da PNIPIC a eles relacionadas.

\section{Formação profissional em PIC para o SUS}

Um primeiro conjunto de estudos que abordam este tema ${ }^{10-14}$ apontam para a falta de preparo de profissionais do SUS para atuar no campo das PIC, cuja formação se mostra deficiente seja na graduação, na pós-graduação ou no âmbito da educação permanente. Estes dados reiteram os dois últimos relatórios de gestão da PNPIC ${ }^{5,6}$, que destacam a formação profissional como um dos principais desafios da política.

Em outros dois artigos aqui incluídos ${ }^{15,16}$ constata-se a ausência de acompanhamento dos profissionais de saúde em relação ao uso de plantas medicinais por usuários. Sabe-se que o conhecimento popular sempre orientou o uso de plantas medicinais sem oferecer grandes riscos à saúde da população. No entanto, dado que o uso incorreto das plantas pode incorrer em alguns prejuízos relacionados à superdosagem, uso de plantas de origem suspeita e erros de prescrição ${ }^{17}$, o acompanhamento do uso de plantas medicinais em casos de usuários com doenças mais graves é desejável.

Além disso, o desconhecimento dos profissionais de saúde sobre as PIC também pode disseminar conceitos equivocados sobre estas terapêuticas, gerando dificuldades na relação médico-paciente e com colegas praticantes dessas especialidades ${ }^{18}$.

Entretanto, é interessante observar como a evidência de uma baixa capacitação profissional em PIC é acompanhada, em alguns dos artigos, pela experiência pessoal de uso de PIC por profissionais do SUS ${ }^{10-13,16,19}$, sendo possível levantar a hipótese de que haja interesse dos mesmos em 


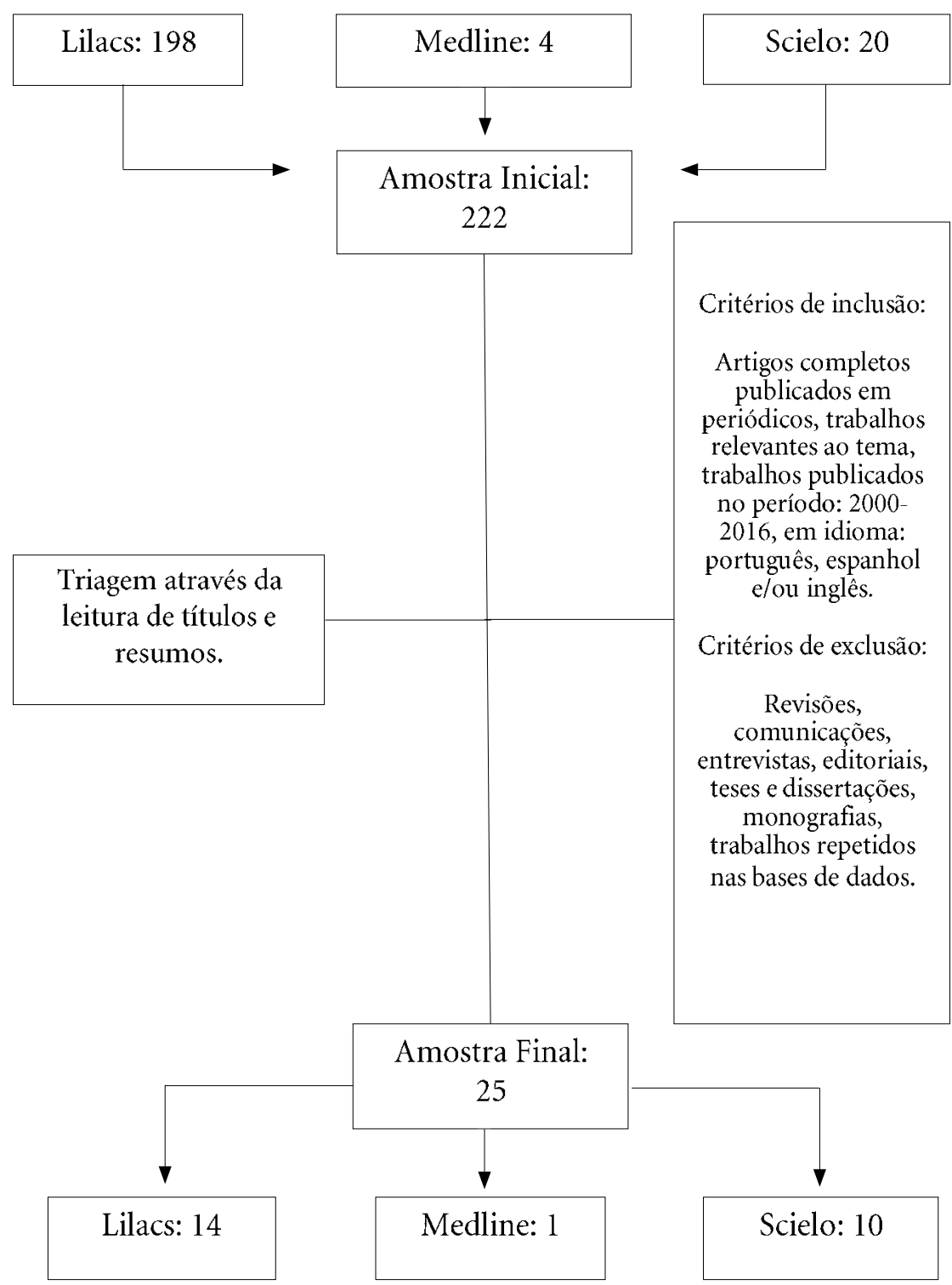

Figura 1. Seleção dos artigos.

se capacitar para incorporar tais práticas em seu trabalho.

Apesar da existência da PNPIC, do aparente baixo nível de capacitação na área e do interesse dos profissionais em se capacitar, a oferta de cursos de formação em PIC que atendam as necessidades do SUS ainda é incipiente no Brasil ${ }^{20,21}$.

De forma geral, a formação em PIC no país se dá no setor privado, por meio de cursos de pósgraduação lato sensu, em programas com cerca de 1.200 horas/aula, capacitando os profissio- nais para prestar provas de títulos de especialista junto aos convênios das entidades de classe. Nos cursos de graduação a grande maioria da oferta se limita a disciplinas eletivas (60hs aula e 4 créditos), sendo ministradas, na maioria das vezes, em poucas universidades por professores especialistas na área, de forma voluntária ${ }^{22}$.

Em um estudo acerca da situação do ensino das PIC em cursos de graduação, Salles et al. ${ }^{21}$, constatou que das 209 instituições públicas de ensino de Enfermagem, Medicina e Fisioterapia 
Quadro 1. Temas, potencialidades e fragilidades de implantação da PNPIC.

\begin{tabular}{|c|c|c|c|}
\hline Tema & Síntese do conhecimento & $\begin{array}{c}\text { Potencialidade/ } \\
\text { Fragilidade }\end{array}$ & Artigos \\
\hline \multirow[t]{3}{*}{$\begin{array}{l}\text { Formação profissional } \\
\text { em PIC para o SUS }\end{array}$} & $\begin{array}{l}\text { Falta de preparo de profissionais do } \\
\text { SUS para atuar no campo das PIC }\end{array}$ & Fragilidade & 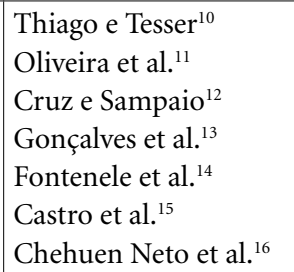 \\
\hline & $\begin{array}{l}\text { Oferta incipiente de cursos de } \\
\text { formação em PIC que atendam as } \\
\text { necessidades do SUS }\end{array}$ & Fragilidade & $\begin{array}{l}\text { Teixeira e } \text { Lin }^{20} \\
\text { Salles et al. }{ }^{21}\end{array}$ \\
\hline & $\begin{array}{l}\text { Experiência pessoal de uso de PIC } \\
\text { por profissionais do SUS }\end{array}$ & Potencialidade & $\begin{array}{l}\text { Thiago e Tesser }{ }^{10} \\
\text { Oliveira et al. }{ }^{11} \\
\text { Cruz e Sampaio }{ }^{12} \\
\text { Gonçalves et al. }^{13} \\
\text { Chehuen Neto et al. }^{16} \\
\text { Ischkanian e Pelicioni }^{19}\end{array}$ \\
\hline \multirow[t]{2}{*}{$\begin{array}{l}\text { Estruturação da oferta } \\
\text { em PIC, acesso e } \\
\text { promoção de saúde }\end{array}$} & $\begin{array}{l}\text { Restrição de acesso às PIC na } \\
\text { atenção secundária }\end{array}$ & Fragilidade & $\begin{array}{l}\text { Santanna et al. }{ }^{23} \\
\text { Lima et al. }^{24} \\
\text { Nagai e Queiroz } \\
\end{array}$ \\
\hline & $\begin{array}{l}\text { PIC na APS favorece ações de } \\
\text { promoção da saúde }\end{array}$ & Potencialidade & $\begin{array}{l}\text { Nagai e Queiroz }{ }^{25} \\
\text { Silva e Tesser }{ }^{26} \\
\text { Cintra e Figueiredo }^{27}\end{array}$ \\
\hline \multirow[t]{4}{*}{$\begin{array}{l}\text { Conhecimento, acesso } \\
\text { e aceitação de usuários } \\
\text { em relação às PIC }\end{array}$} & $\begin{array}{l}\text { Baixo conhecimento e acesso de } \\
\text { usuários às PIC oficiais }\end{array}$ & Fragilidade & $\begin{array}{l}\text { Fontanella et al. }{ }^{28} \\
\text { Rui et al. }{ }^{29} \\
\text { Marques et al. }{ }^{30}\end{array}$ \\
\hline & $\begin{array}{l}\text { Interesse de usuários em relação às } \\
\text { PIC oficiais }\end{array}$ & Potencialidade & $\begin{array}{l}\text { Fontanella et al. }^{28} \\
\text { Rui et al. }{ }^{29}\end{array}$ \\
\hline & $\begin{array}{l}\text { Uso informal de medicina } \\
\text { tradicional }\end{array}$ & Potencialidade & $\begin{array}{l}\text { Oliveira et al. }{ }^{11} \\
\text { Fontanella et al. }^{28} \\
\text { Oliveira e Salvi }{ }^{31} \\
\end{array}$ \\
\hline & $\begin{array}{l}\text { Identidade entre PIC e elementos } \\
\text { das práticas de saúde presentes na } \\
\text { cultura e religião brasileiras. }\end{array}$ & Potencialidade & $\begin{array}{l}\text { Monteiro e Iriart }{ }^{32} \\
\text { Justo e Gomes }\end{array}$ \\
\hline $\begin{array}{l}\text { Conhecimento de } \\
\text { profissionais e gestores } \\
\text { em relação ã PNPIC }\end{array}$ & $\begin{array}{l}\text { Baixo conhecimento de } \\
\text { profissionais e gestores em relação } \\
\text { à PNPIC }\end{array}$ & Fragilidade & $\begin{array}{l}\text { Thiago e Tesser }{ }^{10} \\
\text { Chehuen Neto et al. }^{16} \\
\text { Ischkanian e Pelicioni }^{19} \\
\text { Santos et al. }{ }^{34} \\
\text { Santos e Tesser } \\
\end{array}$ \\
\hline $\begin{array}{l}\text { Escopo, Monitoramento } \\
\text { e Avaliação da PNPIC }\end{array}$ & $\begin{array}{l}\text { Indefinição de escopo da PNPIC } \\
\text { e falhas de registro do SIA-SUS e } \\
\text { CNES. }\end{array}$ & Fragilidade & $\begin{array}{l}\text { Lima et al. }{ }^{24} \\
\text { Sousa et al. } .^{36} \\
\text { Santos et al. }{ }^{37} \\
\end{array}$ \\
\hline
\end{tabular}

contatadas, somente 43 (32,3\%) oferecem disciplinas relacionadas ao tema. Dentre os três cursos, os de Enfermagem são aqueles que mais oferecem disciplinas $(26,4 \%)$, seguido de Medicina $(17,5 \%)$ e Fisioterapia (14,6\%).

Os programas de residência estão disponíveis principalmente para a categoria médica. Apro- vados em 2002, através da Resolução Conselho Federal de Medicina (CFM) $n^{\circ} 1634 / 2002^{38}$, as residências médicas são oferecidas em dois anos (R1 e R2), em nove instituições para a área da acupuntura e em apenas uma instituição para a área de homeopatia ${ }^{21}$. Para os demais profissionais de saúde, a oferta de residências se dá por 
meio das Residências Multiprofissionais em Saúde da Família que raramente oferecem módulos que tratam das PIC ${ }^{21}$.

Imagina-se que a baixa oferta de educação em PIC em consonância com os princípios da Saúde Coletiva deva-se à baixa demanda deste tipo de profissional no SUS, principalmente diante da recente implantação da PNPIC a da falta de financiamento específico para a política.

No entanto, a forma como o movimento da medicina integral foi incorporado no Brasil também é útil na compreensão desta lacuna nas instituições públicas de ensino. Ao retomar a história deste movimento no Brasil, Mattos ${ }^{39}$ relata que diferentemente do que ocorreu nos EUA, a medicina integral não se institucionalizou, permanecendo dentro dos departamentos de medicina preventiva. Neste contexto, o eixo de interpretação da medicina integral se reconfigurou e o problema da fragmentação e do reducionismo da assistência em saúde foi colocado em um plano mais amplo, ligado ao fluxo e organização dos serviços.

Por muito tempo no contexto brasileiro, a discussão sobre a integralidade deixou de privilegiar a boa prática médica para abordar a estruturação dos serviços e das práticas, das políticas e programas especiais, bem como o do acesso dos usuários aos diversos níveis de atenção.

Apesar da lacuna no ensino em PIC, Azevedo e Pelicioni ${ }^{22}$ sinalizam a existência de iniciativas na educação pública que se mostram promissoras. No âmbito da pós-graduação stricto sensu, as autoras destacam os espaços dos laboratórios e grupos de pesquisa em PIC vinculados às universidades, como o Grupo de Racionalidades Médicas sediado na Universidade Federal Fluminense (UFF), o Laboratório de Pesquisas e Práticas de Integralidade em Saúde (Lappis), da Universidade Estadual do Rio de Janeiro (UERJ), o Laboratório de Práticas Alternativas, Complementares e Integrativas em Saúde (Lapacis), na Universidade Estadual de Campinas (Unicamp) e o Grupo de Práticas Complementares de Saúde (GPCS) da Escola de Enfermagem da Universidade de São Paulo (USP).

Já no âmbito da graduação, as autoras chamam a atenção para os recém implantados cursos em Saúde Coletiva/Saúde Pública, como por exemplo o da Universidade de São Paulo (USP) e o da Universidade Federal do Rio de Janeiro (UFRJ), nos quais se espera que o tema das PIC no SUS seja abordado de forma a suprir a lacuna existente e estimular futuros profissionais nesta área.
No campo da educação permanente, o relatório de gestão da Coordenação Nacional de Práticas Integrativas e Complementares ${ }^{5}$ pontua a necessidade de utilização das estratégias do MS viabilizadas pela Política de Educação em Saúde, como: o Sistema Universidade Aberta do SUS (UNA-SUS); o Programa Nacional de Tele Saúde; o Programa de Educação Permanente pelo Trabalho - PET-Saúde; os cursos de especialização e mestrado profissionalizante, entre outros. Com relação às plantas medicinais e fitoterápicos, destacam as iniciativas locais em alguns estados e a inserção de módulos com este tema nos cursos de especialização do MS.

Apesar dos resultados não trazerem dados acerca destes cursos, acredita-se que tais estratégias são de fundamental importância na superação do desafio apontado neste mesmo documento referente a formação e capacitação profissional em número adequado para atuar no SUS, uma vez que oferecem a possibilidade de formação dos profissionais que já atuam no SUS.

\section{Estruturação da oferta em PIC, acesso e promoção de saúde}

Esta categoria reúne estudos que discutem a estruturação das PIC nos serviços e sugerem uma influência do nível de atenção em que se encontra a oferta em PIC sobre o acesso e o potencial destas terapêuticas para promover a integralidade do cuidado através da promoção da saúde.

Um primeiro conjunto de artigos ${ }^{23-25}$ traz relatos de profissionais, usuários e gestores de serviços de nível secundário que apontam para uma restrição do acesso às PIC, com uma alta demanda reprimida, atribuída principalmente ao fato destes atendimentos estarem vinculados apenas à profissão médica.

Este problema parece encontrar solução na estratégia da PNPIC em enfocar ações na APS, tanto pela maior capilaridade da atenção básica quanto pela possibilidade de execução das PIC por profissionais não médicos, mediante o NASF $^{5}$.

Em estudo recente, que analisa a inserção das PIC na APS de grandes cidades brasileiras, os autores assinalam que o modo mais promissor de disseminação da política no SUS é através da Estratégia de Saúde da Família (ESF) de forma conjugada com equipes matriciais. Segundo os autores, estas equipes oferecem retaguarda especializada à APS, atenuam as filas de espera e, dependendo da gestão local, podem matriciar a ESF e realizar atividades grupais no território ${ }^{40}$. 
É importante notar que a aplicação das PIC é regulamentada pelos conselhos de diversas classes profissionais além da médica, a exemplo da acupuntura que pode ser realizada por psicólogos, fisioterapeutas, farmacêuticos, biomédicos, biologistas, enfermeiros e psicólogos, possibilitando ao NASF efetivar estas ações no cenário da APS.

Também nesta categoria, foram inseridos três estudos $^{25-27}$ que trazem relatos de usuários de PIC na APS que apontam para uma apropriação de técnicas e ferramentas voltadas para o autocuidado e mudança no estilo de vida, ou seja, para a promoção da saúde individual.

A concepção mais atual de promoção de saúde se entende a partir de uma perspectiva coletiva cujas ações devem ser realizadas em âmbito político, social, institucional e comunitário, com a finalidade de elaboração e implementação de políticas públicas saudáveis, criação de ambientes favoráveis à saúde, reforço da ação comunitária, desenvolvimento de habilidades pessoais e reorientação do sistema saúde ${ }^{41}$.

Embora a atuação das PIC para promoção de saúde seja comumente localizada no âmbito individual, Tesser ${ }^{42}$ argumenta que esta ação, principalmente quando as PIC são oferecidas em grupos, favorece a socialização e a solidariedade, podendo catalisar um empoderamento coletivo.

Esta potencialidade das PIC é de extrema valia para a PNPIC uma vez que o seu fortalecimento depende da articulação das pessoas que nela atuam, usuários, profissionais e gestores, conforme aponta Barros ${ }^{43}$ que, ao discutir esta questão, atribui à ESF e às PIC o status de movimento social.

Segundo Tesser e Souza ${ }^{44}$, a APS e as PIC são estratégias que portam afinidades eletivas, ou seja, aspectos em comum que se potencializam em um contexto de interação. Por esta razão, os autores defendem que a APS seria o campo mais fértil para a oferta de PIC, uma vez que favorece as ações de promoção de saúde, que constituem o cerne das PIC.

Neste sentido, se a PNPIC possui como justificativa a garantia do princípio da integralidade, o enfoque da política na APS parece ser assertivo, uma vez que amplia as possibilidades de que os usuários sejam ativamente incluídos como sujeitos de seus tratamentos e, ao mesmo tempo, da própria implantação da política.

\section{Conhecimento, acesso e aceitação de usuários em relação às PIC}

Neste tema estão reunidos artigos de natureza exploratória que discutem o conhecimento, acesso e aceitação de usuários do SUS em relação às PIC.

Em consonância com os relatórios de gestão da PNPIC $^{4,5}$ que elencam a divulgação entre os principais desafios da política, três estudos aqui incluídos ${ }^{28-30}$ apontam para um baixo conhecimento e acesso de usuários do SUS em relação às PIC oficializadas pela PNPIC. Vale observar que em dois destes estudos ${ }^{28,29}$ observou-se o interesse dos usuários em conhecer as PIC oficializadas pela PNPIC.

Ao mesmo tempo, constatou-se em três trabalhos $^{11,28,31}$ um acesso informal de usuários a práticas tradicionais de saúde presentes na cultura popular brasileira, em especial o uso de plantas medicinais. Tais práticas, que poderiam ser consideradas como parte da medicina tradicional do Brasil, embora existam em alguns municípios, não foram expressamente incorporadas na PNPIC ${ }^{40}$.

O conhecimento popular das plantas medicinais e de outros recursos terapêuticos de base tradicional são compreendidos como potencialidade no processo de implantação da PNPIC pois, além de representar um patrimônio cultural da população brasileira, são práticas que valorizam a autonomia do usuário no seu processo de cura.

Embora a maioria das PIC não seja original do Brasil, elas carregam uma aproximação ética e filosófica com alguns aspectos essenciais das práticas populares e religiosas de saúde presentes no território nacional, o que é determinante para sua aceitação pela população brasileira.

O estudo de Monteiro e Iriart $^{32}$, por exemplo, argumenta que a aproximação da homeopatia com tais aspectos, como a perspectiva holística, o uso de medicamentos naturais e a escuta aberta e atenta ao paciente favorece a aceitação e incorporação desta PIC pelos usuários, em especial os das classes sociais populares. O estudo de Justo e Gomes $^{33}$, nesta mesma direção, descreve como a implantação da homeopatia na atenção à saúde no município de Santos se consolidou devido ao importante apoio do movimento do espiritismo kardecista, cujos princípios portam afinidades com esta racionalidade médica.

Embora o desconhecimento e o baixo acesso caracterizem uma importante fragilidade da PNPIC, é importante sinalizar que a identidade entre as PIC e muitas das práticas de saúde en- 
raizadas na cultura popular e religiosa do Brasil, também pode ser reconhecida como uma potencialidade do processo de implantação da política pois favorece a legitimação e aceitação destas terapêuticas pela população.

Diante dos diversos empecilhos oferecidos pelas instituições modernas à legitimação das PIC, faz-se necessária a criação de outras estratégias de reconhecimento sócio-institucional destas práticas sendo fundamental neste processo a participação de usuários e profissionais nos espaços de participação política do SUS ${ }^{45}$.

Vale lembrar que a identidade destas terapêuticas perante os organismos internacionais e o governo brasileiro reside justamente no seu pluralismo terapêutico e na alteridade de cuidados, ou seja, na multiplicidade de práticas complementares e tradicionais cuja eficácia clínica e sociocultural se estabeleceu na história e na tradição, antes mesmo da existência da biomedicina ${ }^{46}$.

A estratégia de pluralização terapêutica do SUS, mediante a valorização e oferta das medicinas alternativas e complementares, embora limitada, é defendida por autores da área ${ }^{47}$ pois carrega o potencial de combater a excessiva medicalização do sistema, a despeito da hegemonia política e epistemológica da biomedicina.

\section{Conhecimento de profissionais e gestores do sus em relação à PNPIC}

Os estudos aqui incluídos ${ }^{10,16,19,34,35}$ apontam para um baixo conhecimento de profissionais e gestores do SUS em relação à PNPIC o que constitui uma importante fragilidade da política visto que na ausência de recurso indutor, sua implantação depende em grande parte da participação efetiva destes e dos outros atores do SUS.

A coparticipação de gestores, usuários e profissionais envolvidos nos serviços públicos de saúde é tão fundamental para a implantação da PNPIC quanto os resultados obtidos na assistência, uma vez que permite um planejamento adequado às realidades socioinstitucionais e políticas do território e, consequentemente, a sustentabilidade das ações ${ }^{48}$.

O estudo de Santos e Tesser ${ }^{35}$ sobre a implantação da PNPIC no Recife, retrata com fidelidade esta realidade quando constata como a falta de participação de usuários e a não inclusão de profissionais de saúde no processo de elaboração da política municipal, dificultou a sua consolidação no município que, mesmo após cinco anos de vigor da lei, contava com apenas um serviço funcionando.

\section{Escopo, monitoramento e avaliação da PNPIC}

Atualmente, o monitoramento da PNPIC é realizado a partir dos dados do Sistema de Informações Ambulatoriais do SUS (SIA-SUS), do Sistema do Cadastro Nacional de Estabelecimentos de Saúde (SCNES) e mais recentemente do Sisab/e-SUS, que integra informações específicas das PIC na Atenção Básica. Além disso, são utilizados os resultados do Programa de Melhoria do Acesso e da Qualidade da Atenção Básica (PMAQ-AB), da Pesquisa Nacional em Saúde (PNS), do Cadastro Nacional de PIC e das informações resultantes do apoio institucional ${ }^{48}$.

Os artigos reunidos nesta categoria ${ }^{24,36,37}$ apontam para uma indefinição do escopo da PNPIC que tem por consequência a ocorrência de falhas no registro de informações pertinentes às ações em PIC no Sistema de Informações Ambulatoriais do SUS (SIA-SUS) e no Cadastro Nacional de Estabelecimentos de Saúde (CNES).

Estes achados, corroboram um dos principais desafios apontados pelos relatórios de gestão da política $^{5,6}$, relativo ao Monitoramento e Avaliação.

A falha de registro apontada no estudo de Lima et al. ${ }^{24}$ relaciona-se à existência de diversos recursos terapêuticos que podem ser compreendidos como PIC e que, no entanto, não dispõe de códigos nos formulários do Cadastro Nacional de Estabelecimentos de Saúde (CNES) e no Sistema de Informações Ambulatoriais do SUS (SIA-SUS).

Neste mesmo sentido, o estudo de Sousa et al. ${ }^{36}$ aponta para a existência de duas Portarias ( $\mathrm{n}^{\circ} 971$ de $2006^{49}$ e $^{\circ} 853$ de $2006^{50}$ ) que deixam dúvidas quanto ao termo práticas corporais e outras técnicas, favorecendo diferentes maneiras de registro. Assim como no estudo de Lima et al. ${ }^{24}$, os autores identificaram o registro em separado de práticas não incluídas nos formulários do SIA e do CNES, bem como o registro indevido de muitas práticas não incluídas na PNPIC e/ou tradicionalmente associadas a fatores de risco de natureza biomédica, como: artesanato, caminhada e grupos de prevenção de diabetes, que foram compreendidas pelos gestores como PIC.

Apesar de reconhecer a visibilidade que a política proporcionou às PIC no sistema de informações do SUS, os autores discutem que há uma indefinição do que se compreende como PIC dentro da política, de forma que muitas práticas não estão especificadas no sistema e passam a ser registradas em separado pelos profissionais. 
Outra dificuldade encontrada neste estudo diz respeito ao registro das categorias profissionais que exercem PIC no SUS, cujas nomenclaturas de registro no Código Brasileiro de Ocupações $(\mathrm{CBO})$ não permitem identificar no CNES informações importantes para análise como, por exemplo, se o profissional é de nível médio ou superior ou qual o tipo de atividade que o mesmo realiza no serviço onde está cadastrado.

No estudo de Santos et al. ${ }^{37}$ esta mesma questão foi identificada quando da impossibilidade de discriminar quais eram as profissões dos acupunturistas registrados como não médicos.

A indefinição de escopo da PNPIC favorece um registro inadequado das PIC no SUS e, portanto, dificulta a realização do seu monitoramento e avaliação, fragilizando a consolidação da política.

\section{Considerações finais}

Os temas emergentes na presente revisão visitam alguns dos desafios apontados pelos relatórios de gestão do $\mathrm{MS}^{4,5}$ aprofundando e subsidiando o seu conteúdo.

A necessidade de viabilização da formação e qualificação dos profissionais em número adequado para atuar no SUS é reafirmada. Neste sentido, o uso pessoal de PIC, o possível interesse de profissionais em se capacitar e o número considerável de iniciativas de ensino e pesquisa nas universidades públicas podem catalisar este processo.

Quanto a estruturação dos serviços na rede pública, os dados deste trabalho apontam para a importância de que a mesma aconteça, tal como preconizado na PNPIC, com foco na APS, visto que neste nível de atenção, onde também já estão concentradas a maioria das iniciativas em PIC, estas terapêuticas atingem o seu máximo potencial de ação ao favorecer a promoção de saúde, ampliar a integralidade das ações e a universalização do acesso.

Em relação ao monitoramento e avaliação da política, destaca-se primeiramente a necessidade de revisão do escopo da PNPIC, o que implica em uma definição mais clara das PIC e de quais critérios viabilizam a sua inserção no SUS. Em segundo lugar, são apontados problemas técnicos de registro de informações que sugerem a necessidade de aprimoramento dos sistemas do CNES e do SIA-SUS e de treinamento dos profissionais para operá-los, visando uma maior fidelidade dos dados.
O baixo conhecimento de profissionais e gestores em relação à PNPIC e de usuários em relação às PIC reafirma a necessidade de divulgação. Cabe notar que o uso informal de medicina tradicional pela população e a identidade de valores entre as PIC oficiais e várias tradições presentes na cultura popular brasileira são aspectos fundamentais que devem ser levados em consideração no planejamento das ações de comunicação.

Entretanto, o conhecimento da PNPIC por profissionais, usuários e gestores é apenas um primeiro passo na direção da implantação sustentável da PNPIC, que deve acontecer mediante um processo coletivo de planejamento visando a adequação das ações de assistência às realidades e necessidades loco regionais.

Além disso, como atualmente a PNPIC não dispõe de recurso indutor, torna-se imprescindível a sua defesa política nos conselhos de saúde e outras instâncias do poder público. Se por um lado o marco legal proporcionou uma crescente institucionalização das PIC no SUS, por outro, o enfrentamento dos desafios de implantação da política requer a criação de legislações específicas nos estados e municípios que garantam a destinação de recursos e regulamentem a operacionalização das ações ${ }^{6,27}$.

Outros desafios apontados pelos relatórios de gestão da PNPIC ${ }^{5,6}$, como fornecimento de insumos estratégicos e o investimento em pesquisa e desenvolvimento de produtos não apareceram nos artigos selecionados explicitando um limite da pesquisa.

Diante do cenário de ameaça ao SUS e de seus princípios ético normativos, espera-se que o presente trabalho possa contribuir para o debate acerca da PNPIC e, fundamentalmente, reafirmar a necessidade de articulação política de seus atores para seu avanço.

\section{Colaboradores}

PHL Habimorad trabalhou na pesquisa, concepção e redação final. RS Spagnuolo, KP Patrício e $M$ Demarzo trabalharam na metodologia e redação. FM Catarucci, VHT Bruno, I Beteto trabalharam na redação. V Campolina trabalhou na pesquisa e redação. 


\section{Referências}

1. Justo C. A crise do modelo biomédico e a resposta da promoção de saúde. Rev Portuguesa Saude Publica 2010; 28(2):117-118.

2. Organización Mundial de la Salud (OMS). Estrategia de la OMS sobre medicina tradicional 2002-2005. Genebra: OMS; 2002.

3. Brasil. Ministério da Saúde (MS). Secretaria de Ciência, Tecnologia e Insumos Estratégicos. Departamento de Assistência Farmacêutica. Política Nacional de Plantas Medicinais e Fitoterápicos. Brasília: MS; 2006. (Série B. Textos Básicos de Saúde).

4. Brasil. Ministério da Saúde (MS). Secretaria de Atenção à Saúde. Departamento de Atenção Básica. Política Nacional de Práticas Integrativas e Complementares no SUS - PNPIC-SUS. Brasília: MS; 2006. (Série B. Textos Básicos de Saúde).

5. De Simoni C, Benevides I, Barros NF. As Práticas Integrativas e Complementares no SUS: Realidade e desafios após dois anos de publicação da PNPIC. Rev Bras Saúde Família 2008; (ed. esp.):70-76.

6. Brasil. Ministério da Saúde (MS). Secretaria de Atenção à Saúde. Departamento de Atenção Básica. Relatório de Gestão 2006/2010 Práticas Integrativas e Complementares no SUS. Brasília: MS; 2011.

7. Brasil. Ministério da Saúde (MS). Portaria no 849 , de 29 de março de 2017. Inclui a Arteterapia, Ayurveda, Biodança, Dança Circular, Meditação, Musicoterapia, Naturopatia, Osteopatia, Quiropraxia, Reflexoterapia, Reiki, Shantala, Terapia Comunitária Integrativa e Yoga à Política Nacional de Práticas Integrativas e Complementares. Diário Oficial da União 2017; 28 mar.

8. Brasil. Ministério da Saúde (MS). Portaria no ${ }^{\circ} 702$, de 21 de março de 2018. Altera a Portaria de Consolidação nº 2/GM/MS, de 28 de setembro de 2017, para incluir novas práticas na Política Nacional de Práticas Integrativas e Complementares - PNPIC. Diário Oficial da União 2018; 23 mar.

9. Rother ET. Revisão sistemática $X$ revisão narrativa. Acta paul Enferm 2007; 20(2):V-VI.

10. Thiago SCS, Tesser CD. Percepção de médicos e enfermeiros da Estratégia de Saúde da Família sobre terapias complementares. Rev Saude Publica 2011; 45(2):249-257.

11. Oliveira SGD, Moura FRR, Demarco FF, Nascente PS, Del Pino FAB, Lund RG. An ethnomedicinal survey on phytotherapy with professionals and patients from Basic Care in the Brazilian Unified Health System. J Ethnopharmacol 2012; 140(2):428-37.

12. Cruz PLB, Sampaio SF. O uso de práticas complementares por uma equipe de saúde da família e sua população. Rev APS 2012; 15(4):486-495.

13. Gonçalves RP, Antunes HM, Teixeira JBP, Ludmila OC, Barbosa PR. Profissionais da área da saúde pública: atitudes, conhecimentos e experiências em relação a práticas médicas não convencionais. Rev APS 2008; 11(4):398-405.
14. Fontenele RP, Souza DMP, Carvalho ALM, Oliveira FA. Fitoterapia na Atenção Básica: olhares dos gestores e profissionais da estratégia de Saúde da Família de Teresina (PI), Brasil. Cien Saude Colet 2013; 18(8):2385-2394.

15. Castro MS, Barros NF, Alegre SM, Hoehne EL. O uso de terapia alternativa e complementar por pacientes diabéticos do tipo 2. Brasília Med 2012; 47(1):17-25.

16. Chehuen Neto JA, Sirimarco MT, Duarte Neto JA, Valle DA, Martins JSC, Cândido TC. Uso e compreensão da medicina complementar pela população de Juiz de Fora. HU Rev 2010; 36(4):266-276.

17. World Health Organization (WHO). Consultation on Proper Use of Traditional Medicine and Complementary Medicine by Consumers. Milan, Italy, 1-3 December; 2003. In: Guidelines on developing consumer information on proper use of traditional, complementary and alternative medicine [Internet]. Genebra: WHO; 2004. [acessado 13 Jul 2015]. p. 83. Disponível em: http://apps.who.int/medicinedocs/ pdf/s5525e/s5525e.pdf

18. Franco JA, Pecci C. La relación médico-paciente, la medicina científica y las terapias alternativas. Medicina (Buenos Aires) 2002; 63(2):111-118.

19. Ischkanian PC, Pelicioni MCF. Desafios das práticas integrativas e complementares no SUS visando a promoção da saúde. Rev Bras Crescimento Desenvolv Hum 2012; 22(2):233-238.

20. Teixeira MZ, Lin CA. Educação médica em terapêuticas não convencionais. Rev Med (São Paulo) 2013; 92(4):224-235.

21. Salles LF, Bel Homo RF, Silva MJP. Situação do ensino das práticas integrativas e complementares nos cursos de graduação em enfermagem, fisioterapia e medicina. Cogitare Enfermagem 2014; 19(4):741-746.

22. Azevedo E, Pelicioni MCF. Práticas Integrativas e Complementares de desafios para a educação. Trab. Educ Saude 2011; 9(3):361-378.

23. Santanna C, Hennington EA, Junges JR. Prática homeopática e a integralidade. Interface (Botucatu) 2008; 12(25):233-246.

24. Lima KMV, Silva KL, Tesser CD. Práticas integrativas e complementares e relação com promoção de saúde: experiência de um serviço municipal de saúde. Interface (Botucatu) 2013; 18(49):261-272.

25. Nagai SC, Queiroz MS. Medicina complementar e alternativa na rede básica de serviços de saúde: uma aproximação qualitativa. Cienc Saude Colet 2011; 16(3):1793-800.

26. Silva EDC, Tesser CD. Experiência de pacientes com acupuntura no Sistema Único de Saúde em diferentes ambientes de cuidado e (des) medicalização social. Cad Saude Publica 2013; 29(11):2186-2196.

27. Cintra MER, Figueiredo R. Acupuntura e promoção de saúde: possibilidades no serviço público de saúde. Interface (Botucatu) 2010; 14(32):138-154. 
28. Fontanella F, Speck FP, Piovezan AP, Kulkamp IC. Conhecimento, acesso e aceitação das práticas integrativas e complementares em saúde por uma comunidade usuária do Sistema Único de Saúde na cidade de Tubarão/SC. Arq Catarin Med 2007; 36(2):69-73

29. Rui AAO, Florian MR, Grillo CM, Meirelles MPR, Sousa MLR. Avaliação do conhecimento e interesse em tratamento com Acupuntura na Odontologia pelos usuários do Sistema Único de Saúde dos municípios de Rio Claro e Cerquilho-SP. J Health Sci Inst 2013; 31(3):24-26.

30. Marques LAM, Vale FVR, Nogueira VAS, Mialhe FL, Silva LC. Atenção farmacêutica e práticas integrativas e complementares no SUS: conhecimento e aceitação por parte da população são joanense. Physis 2011; 21(2):663-674.

31. Oliveira AA, Salvi JO. Percepções de acadêmicos de farmácia sobre a homeopatia. Rev Homeopatia 2014; 77(1/2):16-20.

32. Monteiro DA, Iriart JAB. Homeopatia no Sistema Único de Saúde: representações dos usuários sobre o tratamento homeopático. Cad Saude Publica 2007; 23(8):1903-1912.

33. Justo CMP, Gomes MHA. A cidade de Santos no roteiro de expansão da homeopatia nos serviços públicos de saúde no Brasil. Hist Cien Saude Manguinhos 2007; 14(4):1159-1171.

34. Santos FAS, Sousa IMC, Gurgel IGD, Bezerra AFB, Barros NF. Política de práticas integrativas em Recife: análise da participação dos atores. Rev Saude Publica 2011; 45(6):1154-1159.

35. Santos MC, Tesser CD. Um método para implantação e promoção de acesso às Práticas Integrativas e Complementares na Atenção Primária à Saúde. Cien Saude Colet 2012; 17(11):3011-3024.

36. Sousa IMC, Bodstein RCA, Santos FAS, Hortale VA. Práticas integrativas e complementares: oferta e produção de atendimentos em municípios selecionados. Cad Saude Publica 2012; 28(11):2143-2154.

37. Santos FA, Gouveia GC, Martelli PJL, Vasconcelos EMR. Acupuntura no sistema único de saúde e a inserção de profissionais não médicos. Rev Bras Fisioter 2009; 13(4):330-334

38. Conselho Federal de Medicina (CFM). Resolução CFM no 1634 , de 11 de abril de 2002. Dispõe sobre convênio de reconhecimento de especialidades médicas firmado entre o Conselho Federal de Medicina CFM, a Associação Médica Brasileira - AMB e a Comissão Nacional de Residência Médica - CNRM. Diário Oficial da União 2002; 29 abr.

39. Mattos RA. Os sentidos da integralidade: algumas reflexões acerca de valores que merecem ser defendidos. In: Pinheiro R, Mattos RA. Os sentidos da integralidade na atenção e no cuidado à saúde. Rio de Janeiro: UERJ/IMS/Abrasco; 2001. p. 39-64.

40. Souza IMC, Tesser CD. Medicina Tradicional e Complementar no Brasil: inserção no Sistema Único de Saúde e integração com atenção primária. Cad Saude Publica 2017; 33(1):e00150215.
41. Buss PM. Uma introdução ao conceito de promoção da saúde. In: Czeresnia D, Freitas CM, organizadores. Promoção da saúde: conceitos, reflexões, tendências. Rio de Janeiro: Editora Fiocruz; 2003. p. 15-38.

42. Tesser CD. Práticas complementares, racionalidades médicas e promoção de saúde: contribuições pouco exploradas. Cad Saude Publica 2009; 25(8):1732-1742.

43. Barros NF. Abram alas para os novos movimentos sociais: Saúde da Família e Práticas Integrativas e Complementares. Rev Bras Saude Família (Brasilia) 2008; 9(ed. esp.):62-64.

44. Tesser CD, Sousa IMC. Atenção primária, atenção psicossocial, práticas integrativas e complementares e suas afinidades eletivas. Saude Soc 2012; 21(2):336350.

45. Andrade JT, Costa LFA. Medicina Complementar no SUS: práticas integrativas sob a luz da Antropologia médica. Saude Soc 2010; 19(3):497-508.

46. Tesser CD, Barros NF. Medicalização social e medicina alternativa e complementar: pluralização terapêutica do Sistema Único de Saúde. Rev Saude Publica 2008; 42(5):914-920.

47. Galhardi WMP, Barros NF, Leite-Mor ACB. O conhecimento de gestores municipais de saúde sobre a Prática Integrativa e Complementar e sua influência para a oferta de homeopatia no Sistema Único de Saúde local. Cien Saude Colet 2013; 18(1):213-220

48. Brasil. Ministério da Saúde (MS). Secretaria de Atenção à Saúde. Departamento de Atenção Básica. DAB Comunica Boletim PNPIC. Brasília: MS; 2017.

49. Brasil. Ministério da Saúde (MS). Portaria no 971, de 3 de maio de 2006. Aprova a Política Nacional de Práticas Integrativas e Complementares (PNPIC) no Sistema Único de Saúde. Diário Oficial da União; 2006.

50. Brasil. Ministério da Saúde (MS). Portaria no 853, de 17 de novembro de 2006. Identifica integralmente os procedimentos da PNPIC SUS relativos a Medicina Tradicional Chinesa-acupuntura, Homeopatia, Fitoterapia e Práticas Corporais nos Sistemas Nacionais de Informação em Saúde. Diário Oficial da União; 2006.

Artigo apresentado em 18/01/2018

Aprovado em 14/05/2018

Versão final apresentada em 16/05/2018 
